Agrotrópica 28(3): 277 - 284. 2016.

Centro de Pesquisas do Cacau, Ilhéus, Bahia, Brasil

\title{
LEVANTAMENTO DA ENTOMOFAUNA EDÁFICA ASSOCIADA À MATA RIPÁRIA E SISTEMA AGROFLORESTAL, EM RIO BRANCO, AC
}

\author{
Rodrigo Souza Santos ${ }^{1}$, Denise Arruda da Silva², Allana Aryanne Alencar Pereira ${ }^{3}$, Luis Cláudio \\ de Oliveira ${ }^{1}$
}

\begin{abstract}
${ }^{1}$ Embrapa Acre, Rodovia BR 364, km 14, Caixa Postal 321, 69900-970, Rio Branco, Acre, Brasil. rodrigo.s.santos@embrapa.br; luis.oliveira@embrapa.br; ${ }^{2}$ Professora na Escola Prof. Pedro Martinello, Avenida Flaviano Melo, 196, 69908-470, Rio Branco, Acre, Brasil. denise.arrudaa@ hotmail.com; ${ }^{3}$ Professora no Colégio Presbiteriano João Calvino, Avenida Ceará, 2.648, 69900-360, Rio Branco, Acre. Brasil. allana.aryanne@ yahoo.com
\end{abstract}

Insetos são considerados excelentes bioindicadores de mudanças ambientais e da qualidade do ambiente. Estudos de suas comunidades podem ser aplicados no monitoramento ambiental e práticas conservacionistas. As armadilhas de solo constituem-se num método que fornece uma estimativa aproximada do número total de espécies de uma comunidade, além de ser uma metodologia de simples aplicação. O objetivo deste trabalho foi realizar um levantamento da entomofauna edáfica em uma Mata Ripária e Sistema Agroflorestal (SAF), localizados no município de Rio Branco, AC. Foram instaladas 20 armadilhasdo tipo "pitfall trap", os quais consistiram em copos plásticos brancos de $500 \mathrm{~mL}$, equidistantes $5 \mathrm{~m}$ entre si e dispostos em dois transectos, em cada área. Em cada armadilha foi adicionado $250 \mathrm{~mL}$ de solução de formaldeído (1\%) e gotas de detergente líquido neutro. Semanalmente as armadilhas eram recolhidas e o material transportado até o Laboratório de Entomologia da Embrapa Acre, sendo identificados até o nível taxonômico de Ordem. A principal Ordem de insetos encontradas na Mata Ripária e Sistema Agroflorestal foi Hymenoptera, representando $70 \%$ de todos os insetos capturados. As Ordens Hymenoptera, Coleoptera e Diptera foram categorizadas como eudominantes e constantes nas duas áreas.

Palavras-chave: Fauna edáfica, levantamento de insetos, bioindicadores.

\footnotetext{
Survey of soil entomofauna associated with Riparian Forest an Agroforestry System in Rio Branco, Acre state. Insects are considered excellent bioindicators of environmental changes and the quality of the environment. Studies of their communities can be applied in monitoring and conservation practices. The pitfalls traps constitute a method that provides a rough estimate of the total number of species in a community in addition to being a simple methodology. The aim of this work was to carry out a survey of the edaphic fauna in a Riparian Forest and Agroforestry System (AS), located in the county of Rio Branco, Acre, Brazil. Were installed 20 pitfall traps in each area that consisted in white plastic cups of $500 \mathrm{~mL}$ and $5 \mathrm{~m}$ equidistant between them, arranged in two parallel transects in each area. In each trap were added $250 \mathrm{~mL}$ of formaldehyde (1\%) and drops of neutral liquid detergent. Weekly the pitfalls were collected and transported until the Embrapa Acre's Laboratory of Entomology, being identified until the taxonomic level of Order. The main insect Order found in Riparian Forest and Agroforestry System areas was Hymenoptera representing 70\% of all insects collected. The Orders Hymenoptera, Coleoptera and Diptera were categorized as dominant and constant in both areas.
}

Key words: Edaphic fauna, insect survey, bioindicators. 


\section{Introdução}

O processo de expansão da fronteira agropecuária nas últimas décadas, especialmente na região Norte do país, tem resultado em uma crescente fragmentação das florestas nativas. Essas atividades são fontes potenciais de distúrbios sobre o meio ambiente, fundamentalmente quanto às modificações de elementos componentes da estrutura física dos ecossistemas e da paisagem como um todo (Valério Filho, 1995).

Dentre as consequências mais importantes do processo de fragmentação florestal, destacam-se a diminuição da diversidade biológica, o distúrbio do regime hidrológico das bacias hidrográficas, as mudanças climáticas, a degradação dos recursos naturais e a deterioração da qualidade de vida das populações tradicionais (Viana, 1990). Além disto, a fragmentação resulta em remanescentes de vegetação nativa que se avizinham a usos agrícolas e a outras formas de uso, e como resultado o fluxo de radiação, a água e os nutrientes dos solos são alterados significativamente (Saunders et al., 1991; Antonini et al., 2003).

Nesse contexto, a preservação de vegetações do entorno em leitos d'água, lóticos ou lênticos e sistemas de produção mais sustentáveis, são desafios atuais que necessitam de esforços contínuos, a fim de preservar tanto a flora quanto a fauna em um determinado ecossistema, ou agroecossistema. As Matas Ripárias (ciliar e de galeria) desempenham funções ecológicas, sociais e econômicas importantes, auxiliando na conservação do solo contra erosão, empobrecimento, dentre outros (Aquino e Vilela, 2008), promovendo condições ambientais favoráveis. Dentre os sistemas de produção conhecidos, o Sistema Agroflorestal (SAF) é muito utilizado e difundido, principalmente em pequenas propriedades na região Norte do país (Castro et al., 2009). Trata-se de uma forma de uso da terra e manejo dos recursos naturais, nas quais espécies lenhosas (árvores, arbustos, palmeiras etc) são utilizadas em associação com cultivos agrícolas ou animais, na mesma área, de maneira simultânea ou em uma sequência temporal (Montagnini, 1992).

Estes dois ambientes visam preservar as qualidades bióticas e abióticas do solo, proporcionando microclimas favoráveis em termos de umidade, temperatura e incidência solar para o desenvolvimento de uma maior diversidade de organismos (Ferreira e Marques, 1998;
Chabaribery et al., 2008). O solo possui papel importante na manutenção das fontes de água, nível dos rios, na filtragem e armazenamento de água e elementos químicos. Assim, o uso racional do solo garante sua conservação e proteção ambiental garantindo a todos os seres, uma melhor qualidade de vida (Nascimento et al., 2004). Já seu uso indiscriminado resulta na diminuição da densidade $\mathrm{e}$ diversidade de uma vasta quantidade de organismos que habitam o solo e que são fundamentais na decomposição de matéria orgânica e ciclagem de nutrientes (Lima e Serra, 2008).

Com o declínio da qualidade do solo ou o incentivo à sua degradação há redução de biodiversidade animal afetando a manutenção dos ecossistemas terrestres como um todo (Azevedo, 2004). Com a alteração do habitat natural, muitas espécies abandonam o fragmento, sendo que inúmeros indivíduos ou espécies morrem ou são até extintas, até que um novo equilíbrio se estabeleça (Thomazini e Thomazini, 2000).

Os insetos são considerados bons indicadores dos níveis de impacto ambiental, devido à sua grande diversidade de espécies e habitat, além da sua importância, participando dos processos biológicos nos ecossistemas naturais. A Classe Insecta é considerada como a mais evoluída do Filo Arthropoda, abrangendo cerca de $70 \%$ das espécies de animais (Gallo et al., 2002). Filho (1995) relata os insetos como os organismos de maior ocorrência em ambientes florestais. Portanto, o número de Ordens, famílias e espécies destes, diminuem com a elevação do nível de ação antrópica no ambiente (Thomazini e Thomazini, 2002).

Diante da carência de estudos sobre a fauna edáfica no estado do Acre, o presente trabalho teve como objetivo comparar a entomofauna edáfica em duas áreas: uma Mata Ripária e um Sistema Agroflorestal (SAF), no município de Rio Branco, AC.

\section{Material e Métodos}

O estudo foi conduzido em duas áreas: uma Mata Ripária (1003'42.59”'S; 6746’26.48”O) (Figura 1A) e um Sistema Agroflorestal (SAF) (10 03'65.41'S; 6746’42.60”O) (Figura 1B), localizadas no município de Rio Branco, AC, no período de outubro a novembro 

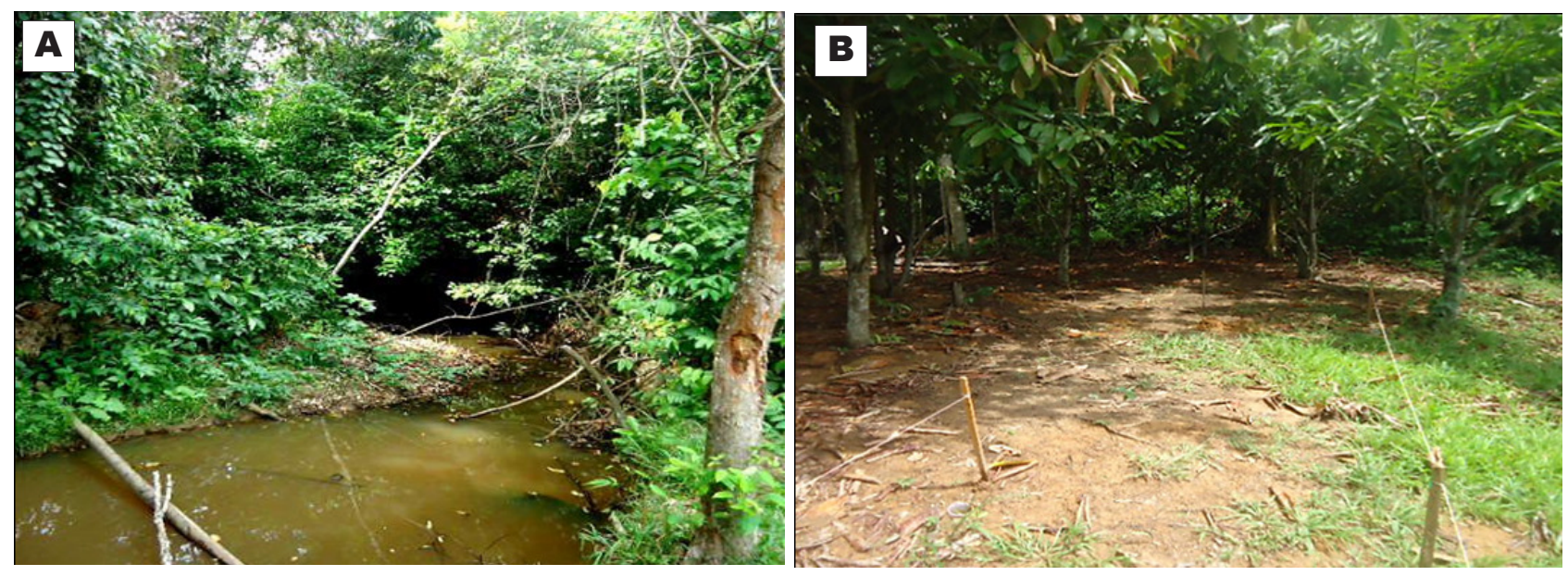

Figura 1: A. Aspecto da vegetação presente na Mata Ripária do Igarapé Santa Maria, Rio Branco, AC. B. Vista dos transectos instalados em Sistema Agroflorestal (SAF).

de 2011. As coletas foram concentradas no período com maior índice pluviométrico na região, o que favorece a ocorrência dos insetos na área.

A Mata Ripária (ciliar) estudada é componente do Igarapé Santa Maria, o qual deságua no Rio Acre. O Sistema Agroflorestal está localizado em um quintal rural (área localizada no perímetro da casa do agricultor), foi implantado em 1998 e, desde sua implantação, não se faz uso de produtos fitossanitários. Este SAF é composto principalmente por espécies frutíferas, tais como: pupunheira, goiabeira, coqueiro, cupuaçuzeiro, cajueiro, bananeira, pitangueira e árvores de grande porte não identificadas.

As duas áreas evidenciam uma forte ação antrópica, caracterizadas por atividades agropecuárias e agrícolas, estradas e pastoreio, principalmente na área de capoeira, vegetação componente da Mata Ripária.

Foram instaladas 20 armadilhas do tipo "pitfall trap" em dois transectos (A e B) em cada uma das áreas de estudo. Os pitfalls consistiram de copos plásticos brancos de $500 \mathrm{~mL}$ identificados, contendo aproximadamente $250 \mathrm{~mL}$ de solução de formaldeído (1\%) e algumas gotas de detergente neutro, a fim de se quebrar a tensão superficial da água e impedir que os animais conseguissem voltar à superfície (Mommertz et al., 1996). O emprego do formaldeído deve-se à sua persistência em temperatura ambiente, bem como sua capacidade de conservação das estruturas dos insetos capturados em condições de campo.

As armadilhas foram distanciadas $10 \mathrm{~m}$ entre si e o distanciamento entre os transectos foi de cinco metros, conforme metodologia adaptada de Santos e Cividanes (2007). Semanalmente as armadilhas eram recolhidas e substituídas por novas. O material coletado era transportado até o Laboratório de Entomologia da Embrapa Acre, onde se procedia a triagem com auxílio de peneira fina. Artrópodes e outros animais que eventualmente eram capturados nas armadilhas eram descartados e não foram contabilizados.

Insetos representantes de cada Ordem sofreram montagem entomológica e os demais foram contabilizados e preservados em via úmida (álcool a 70\%), sendo depositados na Coleção Entomológica da Embrapa Acre. Com auxílio de microscópio estereoscópio, os insetos foram identificados até o nível taxonômico de Ordem, utilizando chaves taxonômicas específicas (Gallo et al., 2002; Thomazini e Thomazini, 2002; Buzzi, 2013). O nível de Ordem foi escolhido a fim de acelerar o processo de triagem e, por esta categoria taxonômica ser suficiente para a caracterização da entomofauna edáfica entre as duas áreas de estudo.

Para determinar as categorias de dominância entre as Ordens, foi utilizada a classificação estabelecida por Friebe (1983):

$$
D \%=\left(\frac{i}{t}\right) \times 100
$$

onde $\mathrm{i}=$ total de indivíduos de uma espécie/Ordem e $\mathrm{t}=$ total de indivíduos coletados, sendo: Eudominante $(D>10 \%)$; Dominante $(D>5<10 \%)$; Subdominante $(D>2 \%<5 \%)$; Eventual $(D=1<2 \%)$ e Rara $(D$ $<1 \%)$. 
A constância foi calculada por meio da porcentagem de ocorrência das Ordens no levantamento, utilizando-se a fórmula:

$$
C=\left(\frac{C i}{N C}\right) \times 100,
$$

em que: $C=$ percentagem de constância; $C i=$ número de coletas contendo a Ordem; $N c=$ número total de coletas efetuadas. Pelas percentagens, as Ordens foram agrupadas em categorias, segundo Silveira Neto et al. (1976) em: 1. Constantes (x) - presentes em mais de 50\% das coletas; 2 . Acessórias (y): presentes entre 25 a $50 \%$ das coletas; 3 . Acidentais (z): presentes em menos de $25 \%$ das coletas.

A diversidade das Ordens foi estimada, utilizandose dos Índices de diversidade de Shannon-Wiener (H') e Simpson (Ds). O Índice de Diversidade de ShannonWiener (H') foi obtido pela fórmula:

$$
H^{\prime}=-\sum p i \log p i,
$$

em que: $p i=$ proporção da espécie em relação ao número total de espécimes/Ordens encontrados nos levantamentos realizados (Pieolou, 1975).

O Índice de Diversidade Simpson (Ds) foi calculado com base na fórmula:

$$
I s=\sum \frac{n i(n i-1)}{N(N-1)}
$$

em que: $I s=$ Diversidade de Simpson; $n i=$ número de indivíduos de cada espécie/Ordem; $N=$ número total de indivíduos (Simpson, 1949).

Foi utilizado o teste T de Student descrito por Poole (1974), através do software PAST 3.01 (Hammer et al., 2001).

\section{Resultados e Discussão}

Foi capturado um total de 2.295 espécimes nas duas áreas estudadas, sendo $1.440(62,7 \%)$ no Sistema Agroflorestal e 855 (37,3\%) na Mata Ripária. Foram coletados insetos representantes de oito Ordens: Hymenoptera, Coleoptera, Blattodea, Orthoptera, Hemiptera, Diptera, Isoptera e Lepidoptera durante o período de estudo (Tabela 1).

As principais Ordens encontradas nas duas áreas foram Hymenoptera (predominantemente formigas), correspondendo a 70,8\% de todos os insetos capturados, seguida por Coleoptera $(11,8 \%)$ e Isoptera $(11,7 \%)$. As demais Ordens juntas corresponderam a apenas 5,7\% do total de espécimes capturados (Tabela 1). Hymenoptera (1.625) e Lepidoptera (2) contribuíram com as maiores e menores quantidades de indivíduos capturados nas armadilhas, respectivamente (Tabela 1). Além de insetos, foram capturados espécimes de Chilopoda, Diplopoda, aranhas e opiliões nos pitfalls, os quais não foram contabilizados.

O número de Ordens foi muito semelhante entre as duas áreas de estudo, com exceção de Lepidoptera, a qual foi encontrada somente na Mata Ripária. Esta similaridade provavelmente está relacionada à proximidade entre as áreas, o que possibilita o fluxo contínuo de insetos entre elas, além do número reduzido de coletas. Os insetos da Ordem Lepidoptera, por não terem o hábito de caminhar no solo, são capturados em menor quantidade em levantamentos por armadilhas do tipo pitfall, as quais são utilizadas frequentemente para estudos de insetos edáficos (Teixeira, 2012).

\begin{tabular}{|c|c|c|c|c|c|c|}
\hline \multirow{2}{*}{ Ordens } & \multicolumn{4}{|c|}{ Local de coleta } & \multirow{2}{*}{ Total } & \multirow{2}{*}{$\%$ do Total } \\
\hline & Mata Ripária & \% de ocorrência & Sistema Agroflorestal & \% de ocorrência & & \\
\hline Hymenoptera & 628 & 73,5 & 997 & 69,2 & 1.625 & 70,8 \\
\hline Coleoptera & 106 & 12,4 & 165 & 11,5 & 271 & 11,8 \\
\hline Blattodea & 04 & 0,5 & 03 & 0,2 & 07 & 0,3 \\
\hline Orthoptera & 06 & 0,7 & 53 & 3,7 & 59 & 2,6 \\
\hline Hemiptera & 03 & 0,4 & 02 & 0,1 & 05 & 0,2 \\
\hline Diptera & 17 & 2,0 & 40 & 2,80 & 57 & 2,5 \\
\hline Isoptera & 89 & 10,4 & 180 & 12,5 & 269 & 11,7 \\
\hline Lepidoptera & 02 & 0,2 & 0 & 0 & 02 & 0,1 \\
\hline Total & 855 & 100 & 1.440 & 100 & 2.295 & 100 \\
\hline
\end{tabular}

Tabela 1. Ordens, número de indivíduos e porcentagem de ocorrência de insetos em Mata Ripária e Sistema Agroflorestal (SAF), em Rio Branco, AC, no período de outubro a novembro de 2011 
Em relação à dominância das Ordens registradas, Hymenoptera, Coleoptera e Isoptera foram categorizadas como eudominantes, tanto na Mata Ripária como no Sistema Agroflorestal. A Ordem Orthoptera e Diptera foram categorizadas como subdominantes no Sistema Agroflorestal e como rara e eventual na Mata Ripária, respectivamente. As Ordens Blattodea, Hemiptera e Lepidoptera foram categorizadas como raras para os dois ambientes (Tabela 2).

Na Mata Ripária e no Sistema Agroflorestal, as Ordens Hymenoptera, Coleoptera, Orthoptera, Diptera e Isopera foram categorizadas como constantes. $\mathrm{Na}$ Mata Ripária, Blattodea e Lepidoptera foram categorizadas como acessórias e Hemiptera como acidental. No Sistema Agroflorestal, as Ordens Blattodea e Hemiptera foram consideradas acessórias (Tabela 2).

As Ordens Hymenoptera, Diptera e Coleoptera são comumente dominantes em trabalhos desta natureza (Oliveira et al., 2013). A Ordem Diptera é considerada megadiversa (Marinoni e Ganho, 2003), enquanto Hymenoptera, especialmente as formigas, são abundantes e ocupam os mais diversos tipos de ambientes (Alencar et al., 2007). Já Coleoptera é a maior Ordem dentro de Insecta, abrigando uma enorme quantidade de espécies que ocupam os mais diversos nichos ecológicos e apresentam grande diversidade de hábitos alimentares (Marinoni e Ganho, 2003).

A análise faunística das Ordens coletadas neste estudo demonstrou, para o Sistema Agroflorestal, um índice de Shannon-Wiener no valor de $\mathrm{H}^{\prime}=1,0$ e Ds = 0,51 para Simpson, enquanto que, para a Mata Ripária o índice Shannon-Wiener foi de $\mathrm{H}^{\prime}=0,89$ e Ds $=0,56$ para Simpon. Estes resultados demonstram uma pequena diferença entre as áreas, principalmente no que tange à densidade populacional de indivíduos/ Ordem, a qual foi superior no Sistema Agroflorestal. Estes valores indicam baixa diversidade de Ordens nestes ambientes, visto que, quanto mais alto é o valor destes índices, maior é a dominância e menor a diversidade (Ribeiro, 2005), os quais combinam riqueza com uniformidade e atribuem maior peso para as espécies raras (Ferraz et al., 2009).

Representantes da Ordem Hymenoptera foram capturados em todo o período de amostragem nas duas áreas. Estes resultados podem estar relacionados ao fato de que, alguns representantes desta Ordem, especialmente as formigas, são dominantes nos ecossistemas tanto pela riqueza de espécies quanto pelo número de indivíduos (Silva et al., 2006) e são facilmente coletadas pelo hábito de forragear em bando (Vieira e Hosokawa, 1989; Ribeiro, 1993). Alguns autores também apontam o predomínio da Ordem Hymenoptera em levantamentos de insetos edáficos (Guariento, 2005; Silva et al., 2006; Santos e Zequi, 2010). O resultado obtido neste levantamento concorda com os resultados de outros trabalhos, evidenciando os himenópteros, especialmente as formigas, os insetos edáficos mais abundantes. Este grupo é um dos maiores dentro de Insecta, compreendendo as vespas, abelhas e formigas. As atividades desses organismos consistem na escavação e/ou ingestão e transporte de material mineral e orgânico no solo, levando à criação de estruturas biogênicas, as quais influem na agregação, propriedades hidráulicas, dinâmica da matéria orgânica

Tabela 2: Constância e Dominância de Ordens de insetos coletados em Mata Ripária e Sistema Agroflorestal (SAF), em Rio Branco, AC, no período de outubro a novembro de 2011

\begin{tabular}{lccccc}
\hline \multirow{2}{*}{ Ordens } & \multicolumn{2}{c}{ Mata Ripária } & & \multicolumn{2}{c}{ Sistema Agroflorestal } \\
\cline { 2 - 3 } \cline { 5 - 6 } & Constância (\%) & Dominância (\%) & & Constância (\%) & Dominância (\%) \\
\hline Hymenoptera & $100 \mathrm{x}$ & 73,45 & & $100 \mathrm{x}$ & 69,23 \\
Coleoptera & $100 \mathrm{x}$ & 12,39 & & $100 \mathrm{x}$ & 11,46 \\
Blattodea & $40 \mathrm{y}$ & 0,47 & & $40 \mathrm{y}$ & 0,21 \\
Orthoptera & $100 \mathrm{x}$ & 0,70 & & $100 \mathrm{x}$ & 3,68 \\
Hemiptera & $20 \mathrm{z}$ & 0,35 & & $40 \mathrm{y}$ & 0,14 \\
Diptera & $60 \mathrm{x}$ & 2,00 & & $80 \mathrm{x}$ & 2,78 \\
Isoptera & $100 \mathrm{x}$ & 10,41 & & $80 \mathrm{x}$ & 12,50 \\
Lepidoptera & $40 \mathrm{y}$ & 0,23 & & - & - \\
\hline
\end{tabular}

As letras minúsculas após os valores significam: $\mathrm{x}$ - Ordem constante; y - Ordem acessória; z - Ordem acidental. 
e na composição, abundância e diversidade de outros organismos do solo (Lavelle e Spain, 2001).

Segundo Brown (1997), os insetos menores são mais sensíveis à ação antrópica, diminuindo ou até desaparecendo após uma perturbação. Neste contexto, citam-se os insetos de solo e serrapilheira, como microlepidópteros, microhimenópteros, besouros detritívoros e pequenas formigas. Este mesmo autor afirma que os indivíduos ou espécies das Ordens Orthoptera, Hemiptera, Diptera, Lepidoptera, Hymenoptera e Coleoptera são os mais importantes bioindicadores.

A ação antrópica nas áreas estudadas também pode ser um fator que desempenha influência na comunidade de insetos, principalmente as formigas. De acordo com Lima et al. (2003), espécies de formigas são os organismos pioneiros na colonização e ocupação de áreas antropizadas.

Faz-se necessária a realização de estudos sistemáticos de levantamento da entomofauna edáfica na região amazônica, uma vez que há notável carência de trabalhos de cunho semelhante, dificultando uma compreensão holística da biodiversidade nos diversos ecossistemas amazônicos. Por este motivo, torna-se imprescindível um maior esforço no conhecimento da prevalência destes insetos que, muitas vezes são ignorados, apesar de sua importância na regulação e manutenção do equilíbrio ecológico.

\section{Conclusão}

As Ordens Hymenoptera, Coleoptera e Diptera foram categorizadas como eudominantes e constantes, tanto na Mata Ripária como no Sistema Agroflorestal. Hymenoptera correspondeu a mais de $70 \%$ de todos os espécimes capturados no levantamento.

\section{Literatura Citada}

ALENCAR, I. D. C. C. et al. 2007. Perfil da fauna de vespas parasitóides (Insecta, Hymenoptera) em uma área de Mata Atlântica do Parque Estadual de Pedra Azul, Domingos Martins, Espírito Santo, Brasil. Arquivos do Instituto Biológico (Brasil) 74 (2): 111-114.

Agrotrópica 28(3) 2016
ANTONINI, Y. et al. 2003. Insetos. In: Rambaldi, D. M.; Oliveira, D. A. S. de. orgs. Fragmentação de ecossistemas: causas, efeitos sobre a biodiversidade e recomendações de políticas públicas. Brasília: MMA/SBF. 324p.

AQUINO, F. G.; VILELA, M. F. 2008. Importância das matas ripárias. Disponível em: <http:// www.infobibos.com/Artigos/2008_4/matas/ index.htm> Acesso em: 03 out. 2016.

AZEVEDO, A. C. de. 2004. Funções ambientais do solo. In: Azevedo, A. C. de; Dalmolin, R. S. D.; Pedron, F. de A. orgs. Fórum Solos e ambiente, 1. Santa Maria, Pallotti. pp.7-22.

BROWN, K. S. 1997. Insetos como rápidos e sensíveis indicadores de uso sustentável de recursos naturais. In: Martos, H. L.; Maia, N. B. Indicadores Ambientais. pp.143-151.

BUZZI, Z. J. 2013. Entomologia didática. Curitiba, PR, UFPR. 579p.

CASTRO, A. P. de. et al. 2009. Os sistemas agroflorestais como alternativa de sustentabilidade em ecossistemas de várzea no Amazonas. Acta Amazônica (Brasil) 39 (2): 279-288.

CHABARIBERY, D. et al. 2008. Avaliação do processo de implantação de projetos demonstrativos para recuperação de matas ciliares do Estado de São Paulo. Revista de Economia Agrícola (Brasil) 55 (1): 89-105.

FERRAZ, A. C. P.; GADELHA, B. Q.; AGUIARCOELHO, V. M. 2009. Análise faunística de Calliphoridae (Diptera) da Reserva Biológica do Tinguá, Nova Iguaçu, Rio de Janeiro. Revista Brasileira de Entomologia (Brasil) 53 (4): 620-628.

FERREIRA, R. L.; MARQUES, M. M. G. S. M. 1998. A fauna de artrópodes de serapilheira de áreas de monocultura com Eucalyptus sp. e mata secundária heterogênea. Anais Sociedade Entomológica Brasil 27 (3):395-403.

FILHO, E. B. 1995. Cupins e florestas. In: Filho, E. B.; Fontes, L. R. orgs. Alguns aspectos atuais da biologia e controle de cupins. Piracicaba, SP, FEALQ. pp. 127-140. 
FRIEBE, B. 1983. Zur Biologie eines Buchenwaldbodens: 3. Die kaferfauna. Carolinea 41:45-80.

GALLO, D. et al. 2002. Entomologia Agrícola. Piracicaba, SP, FEALQ. 920p.

GUARIENTO, H. F. 2005. Levantamento entomológico na Fazenda Prata, São João da Boa Vista, SP. São João da Boa Vista, UNIFEOB. 42p. (Monografia. Graduação em Ciências Biológicas).

HAMMER, O.; HARPER, D. A. T. ; RYAN, P. D. 2001. PAST: Paleontological Statistics software package for education and data analysis. Palaeontologia Electronica 4 (1):1-9.

LAVELLE, P.; SPAIN, A. V. 2001. Soil ecology. Amsterdam, Kluwer Scientific. 678p.

LIMA, A. A. de; LIMA, W. L. de; BERBARA, R. L. L. 2003. Diversidade da mesofauna de solo em sistemas de produção agroecológica. In: Congresso Brasileiro de Agroecologia, 1. Porto Alegre, RS. Anais. Porto Alegre, RS, EMATER/ RS-ASCAR.

LIMA, V. P.; SERRA, A. L. 2008. Análise morfológica comparada da venação de asas da ordem Diptera (Linnaeus, 1758, Arthropoda, Insecta). ConScientiae Saúde (Brasil) 7 (4):525-533.

MARINONI, R. C.; GANHO, N. G. 2003. Fauna de Coleoptera no Parque Estadual de Vila Velha, Ponta Grossa, Paraná, Brasil. Abundância e riqueza das famílias capturadas através de armadilhas de solo. Revista Brasileira de Zoologia (Brasil) 20 (4): 37-744.

MOMMERTZ, S. et al. 1996. A comparison of D-vac suction, fenced and unfenced pitfall trap sampling of epigeal arthropods in agroecosystems. Annales Zoologici Fennici 33:117-124.

MONTAGNINI, F. 1992. Mixed tree plantations: experiments with native trees in Costa Rica and Argentina. Agroforestry Today 4 (3):4-5.

NASCIMENTO, P. C.; GIASSON, E.; JÚNIOR, I. A. V. 2004. Aptidão de uso dos solos e meio ambiente. In: Azevedo, A. C.; Dalmolin, R. S. D.; Pedron, F. de A. eds. Solos \& Ambiente - I Fórum. Santa Maria, RS, Pallotti. pp.41-57.
OLIVEIRA, I. B. R. et al. 2013. Diversidade da entomofauna em uma área de Caatinga no município de Bom Jesus-PI, Brasil. Científica (Brasil) 41 (2): 150-155.

PIEOLOU, E. C. 1975. Ecological diversity. New York: John Wiley \& Sons. 165p.

POOLE, R. W. 1974. An introduction to quantitative ecology. New York: McGraw-Hill. 480p.

RIBEIRO, F. V. 2005. Biodiversidade e distribuição geográfica de Anastrepha spp. (Diptera: Tephritidae) no Alto e Médio Rio Solimões, Amazonas. Dissertação Mestrado. Manaus, AM, FCA/UFAM. 106p.

RIBEIRO, J. D. 1993. Influência do folheto e clareiras na ocorrência de formigas atraídas por iscas em floresta tropical da Amazônia Central. In Reunião Anual da Sociedade Brasileira Para o Progresso da Ciência, 45, Recife, Pernambuco. Recife, PE, SBPC. 569p.

SANTOS, A. A.; ZEQUI, J. A. C. 2010. Entomofauna da floresta Doralice, Ibiraporã Paraná, Brasil, coletada em armadilha de solo. Terra e Cultura (Brasil) 51:91-99.

SANTOS, R. S.; CIVIDANES, F. J. 2007. Registro de opiliões (Arachnida: Opiliones) em três agroecossistemas e remanescente florestal. Ecossistema (Brasil) 32 (1,2): 9-12.

SAUNDERS, D. A.; HOBBS, R. J.; MARGULES, C. R. 1991. Biological consequences of ecosystem fragmentation: a review. Conservation Biology 5 (1):18-35.

SILVA, R. A. et al. 2006. Levantamento de insetos no cerrado amapaense. In Reunião Anual do Instituto Biológico ,19. São Paulo, SP, RAIB. 3p.

SILVEIRA NETO, S.; NAKANO, O.; VILA NOVA, N. A. 1976. Manual de ecologia dos insetos. Piracicaba, SP, Ceres. 419p.

SIMPSON, E. H. 1949. Measurement of diversity. Nature 163:688.

TEIXEIRA, F. M. 2012. Técnicas de captura de Hymenoptera. Vértices (Brasil) 14 (1):169-198.

THOMAZINI, M. J.; THOMAZINI, A. P. B. W. 2000. A fragmentação florestal e a diversidade 
de insetos nas florestas tropicais úmidas. Rio Branco, AC, EMBRAPA ACRE. Documentos n. 57.21p.

THOMAZINI, M. J.; THOMAZINI, A. P. B. W. 2002. Levantamento de insetos e análise entomofaunística em floresta, capoeira e pastagem no sudeste acreano. Rio Branco, AC, EMBRAPA ACRE. Boletim de Pesquisa e Desenvolvimento n. 35. 41p.

VALÉRIO FILHO, M. 1995. Gerenciamento de bacias hidrográficas com aplicação de técnicas de geoprocessamento. In: Tauk-Tornisielo, S. M.;
Gobbi, N.; Foresti, C. orgs. Análise ambiental: estratégias e ações. São Paulo, SP, Fundação Salim Farah Maluf. pp. 135-140.

VIANA, V. M. 1990. Biologia e manejo de fragmentos florestais naturais. In: Congresso Florestal Brasileiro, 6. Anais. Campos do Jordão, SP, SBS/ SBEF. pp. 113-118.

VIEIRA, O.; HOSOKAWA, R. T. 1989. Composição florística da vegetação da regeneração natural. 1 ano após diferentes níveis de exploração de uma floresta tropical úmida. Acta Amazônica (Brasil) 19: 401-413. 